\title{
BMJ Open Resuscitation in hip fractures: a systematic review
}

To cite: Rocos B,

Whitehouse MR, Kelly MB. Resuscitation in hip fractures: a systematic review. BMJ Open 2017;7:e015906. doi:10.1136/ bmjopen-2017-015906

- Prepublication history and additional material are available. To view these files please visit the journal online (http://dx.doi.org/ 10.1136/ bmjopen-2017-015906).

Received 13 January 2017 Revised 27 February 2017 Accepted 28 February 2017

\section{CrossMark}

${ }^{1}$ Trauma and Orthopaedics, North Bristol NHS Trust, Bristol, UK

${ }^{2}$ Musculoskeletal Research Unit, University of Bristol, Bristol, UK ${ }^{3}$ Trauma and Orthopaedics, North Bristol NHS Trust, Bristol, UK

Correspondence to

Brett Rocos;

brett.rocos@bristol.ac.uk

\section{ABSTRACT}

\section{OBJECTIVES}

To evaluate the evidence for the resuscitation of patients with hip fracture in the preoperative or perioperative phase of their treatment and its impact on mortality. Design We searched MEDLINE, EMBASE, CENTRAL and PROSPERO databases using a systematic search strategy for randomised trials and observational studies investigating the fluid resuscitation of any patient with hip fracture. No language limits were applied to the search, which was complemented by manually screening the reference lists of appropriate studies.

Outcome measures Mortality at 1 week, 30 days and 1 year following surgery.

Results Two hundred and ninety-eight citations were identified, and 12 full manuscripts were reviewed; no studies satisfied the inclusion criteria. The background literature showed that the mortality for these patients at 30 days is approximately $8.5 \%$ and that bone cement implantation syndrome is insufficient to explain this. The literature was explored to define the need for an interventional investigation into the preoperative resuscitation of patients with hip fracture.

Conclusions Patients with hip fracture show similar physiological disturbance to major trauma patients. Nineteen per cent of patients presenting with hip fracture are hypoperfused and $50 \%$ show preoperative anaemia suggesting that under resuscitation is a common problem that has not been investigated. A properly conducted interventional trial could improve the outcome of these vulnerable patients.

\section{INTRODUCTION}

\section{The scale of the problem}

Across England, 65000 people suffer a hip fracture every year, with a cost to the National Health Service of approximately $£ 2$ billion. ${ }^{1}$ While some of these fractures occur in younger patients, the majority occur in elderly adults, with a mean age at presentation of 80 years. A best practice tariff for fragility hip fractures was introduced in 2010 with the aim of encouraging prompt surgery and appropriate involvement of geriatric medicine specialists. $^{2}$ Despite the improvements in the care of patients with hip fractures since then, the diagnosis is still considered by some to be a preterminal event with a 30-day mortality of $8 \%$ (equating to over 5000 deaths per

\section{Strengths and limitations of this study}

- This review addresses a significant public health disease with a very poor prognosis.

- Thorough literature search revealed no relevant articles.

- Current practice has no evidence base and requires a clinical trial to inform it.

- A small improvement in mortality would lead to a large real number of patients surviving.

year) and a 1-year mortality of approximately $20 \%$. $^{34}$

The care pathway of these patients is complicated. Patients are typically frail and generally have multiple comorbidities, polypharmacy and are often living dependently at the time of injury. ${ }^{3-8}$ Forty per cent shows cognitive impairment and 20\% will present with an acute delirium. Despite these data suggesting that a hip fracture is a potentially devastating event with multiple factors influencing overall outcome and survival, this population continues to be managed as if the hip fracture was an isolated, skeletal injury.

In contrast, patients who sustain major trauma or multiple fractures, where multiple pathologies influence outcomes, are routinely managed in a secondary and tertiary trauma network with multidisciplinary input and aggressive resuscitation strategies. In recent years, this has led to an improvement in their outcomes and a marked reduction in mortality. ${ }^{9}$

It has been established that hip fracture in elderly adult patients causes a similar physiological insult as major trauma does in younger patients and so should be considered equivalent to major trauma. ${ }^{10} 11$ For young patients who sustain major trauma, the primary early cause of death is shock, which modern protocols tackle with early aggressive fluid resuscitation. ${ }^{12}$ Furthermore, as a result of military practices, modern civilian trauma protocols dictate that patients who need intravenous resuscitation on the assumption of shock in major trauma are 
resuscitated with blood rather than crystalloid or colloid fluids prior to the receipt of laboratory or point of care testing results. ${ }^{13-17}$

A hip fracture, in an elderly, vulnerable patient, constitutes a major traumatic event. While definitive evidence is absent, it is strongly suggested that from the work in patients who have sustained major traumatic injuries that patients who are under resuscitated have worse outcomes and that resuscitation is most effective when it is performed with blood rather than crystalloid or colloid fluids. ${ }^{13-15} 1819$ It then follows that early and appropriate resuscitation in the elderly hip fracture population, tempered by attention to their fragile cardiovascular system, has the potential to improve survivorship and perhaps overall outcome.

We have conducted a literature review to identify relevant evidence supporting the use of blood resuscitation in adult patients aged 65 years and over who have presented within 24hours of sustaining a fracture of the proximal femur when compared with crystalloid or colloid resuscitation. Furthermore, we describe our experience and results of performing a pilot study investigating this intervention, a patient's perspective of the clinical problem and present strategies used in initiating and maintaining the investigation.

\section{METHODS}

The first step in conducting the investigation was a review of relevant existing evidence. To conduct a comprehensive study of the evidence, we performed a systematic review with narrative review of the literature. Our review was conducted in accordance with preferred reporting for systematic reviews and meta-analyses (PRISMA) guidelines. ${ }^{20}$

No protocol was registered for the review because of its small size. We then identified a precise population, intervention, comparator, outcome (PICO) question. In this review, we stipulated the question as: in patients aged $\geq 65$ years presenting with fracture of the proximal femur who are resuscitated with blood products compared with crystalloid, colloid or other intravenous fluid prior or during surgery, are 30-day mortality and morbidity rates reduced?

Using the OVID portal (Wolters Kluwer, Alphen aan den Rijn, The Netherlands), the MEDLINE (1950present), EMBASE (1980-2015 week 16), PROSPERO (no date limits) and CENTRAL (no date limits) databases were searched for articles using a systematic search strategy. The search defined the population as those with hip fracture and mapped this to Medical Subject Headings (MeSH) terms and defined the intervention as fluid administration and/or resuscitation with this and synonyms mapped to MeSH (online supplementary appendix 1). No language limits were placed on the searches. We complemented the search by manually scanning reference lists of identified articles for relevant publications.
The titles of articles identified by the search strategy were then screened by a single author (BR) to verify inclusion into the review. The abstracts of titles that showed potential for inclusion were then reviewed by a single author (BR). For those papers where the abstract was insufficient to confidently either include or exclude from the review, the full manuscript was sourced (figure 1). Any ambiguity for inclusion was resolved through discussion between all authors. Data were to be collected through a custom designed data collection tool by a single author (BR). Bias was to be assessed through the same tool, and its influence included within the narrative analysis. The assessment of bias was based on the criteria laid out by the Critical Appraisal Skills Programme. ${ }^{21} 22$

Outcomes analysed were to include unadjusted 30-day mortality and length of stay as a surrogate for function and morbidity. Summary measures anticipated to be used were ORs. Any assessment of health economics would also be analysed. Subgroup analysis of the very elderly patients (aged $\geq 80$ years) would be performed in the event that these data were available.

\section{RESULTS}

Despite widespread focus on the care in this population in the developed world, a systematic search of the literature shows that preoperative resuscitation in the frail elderly patient with hip fracture has been overlooked. The search did not yield any articles that investigated fluid resuscitation or the use of blood as a fluid for resuscitation prior to surgery in adults aged $\geq 65$ years presenting with hip fracture, and none assessed the impact of modifying preoperative fluid resuscitation on mortality or functional outcomes in this population. References to fluid resuscitation focused on anaesthetic techniques and the monitoring of fluid administration. Most articles do not identify preoperative resuscitation in these patients as an opportunity to modify outcomes. The existing literature focuses on blood transfusion (as opposed to blood resuscitation) as a treatment for established anaemia or investigates techniques and strategies for controlling the postoperative administration of intravenous fluid.

Inadequate resuscitation is easily missed in this patient group and would be rapidly and safely treated in any other age group with the same relative traumatic insult; however, standard practice in hip fracture care is to maintain the existing fluid status of patients using intravenous saline. ${ }^{12}{ }^{23-26}$ While the literature does not specifically investigate resuscitation in hip fracture, it does offer some supporting evidence for early blood product resuscitation in this vulnerable group.

The blood loss associated with a hip fracture has been quantified. Kumar et al showed that the average haemoglobin concentration fell by $2.23 \mathrm{~g} / \mathrm{dL}$ in subtrochanteric fractures, $1.1 \mathrm{~g} / \mathrm{dL}$ in intertrochanteric fractures and $0.7 \mathrm{~g} / \mathrm{dL}$ in intracapsular fractures following admission but before surgery. Foss et al suggested that this only worsens with treatment due to blood loss during 


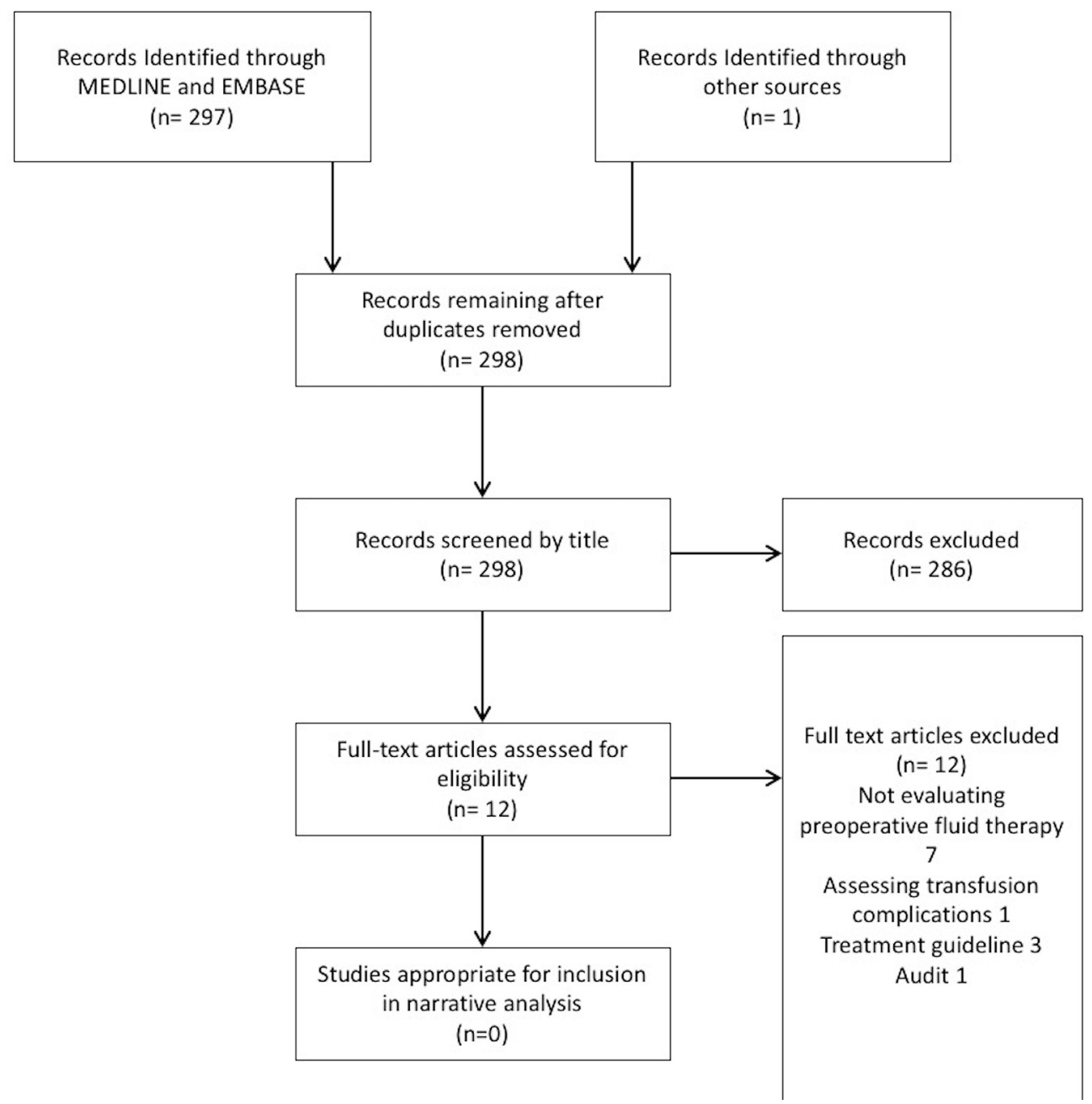

Figure 1 The preferred reporting for systematic reviews and meta-analyses (PRISMA) diagram representing the systematic literature search.

hip fracture surgery ranging from 547 to $1473 \mathrm{~mL}{ }^{27}{ }^{28}$ Irrison et al showed that the haemoglobin concentration follows a linear decline in the first 3 days postoperatively, suggesting that measuring haemoglobin concentration 24 hours will not detect incomplete postoperative resuscitation. ${ }^{29}$

Inadequate resuscitation leads to a relative hypovolaemia that is likely to have two fundamental consequences: hypoperfusion (shock) and reduced oxygen carrying capacity, which exacerbates the effects of the hypoperfusion. Overt shock in hip fracture is uncommon in the absence of other injuries. Occult shock (ie, shock not evident on examination or non-invasive investigation) is defined by a serum lactate higher than $2.5 \mathrm{mmol} / \mathrm{L}$. It is associated with a significant increase in the risk of death, is more common than overt shock and is under diagnosed. ${ }^{23}{ }^{30-35}$ Uzoigwe et al showed that there is a 1.2-fold increase in the odds of death per $1 \mathrm{mmol} / \mathrm{L}$ increase in serum lactate in elderly hip fracture patients, a trend also observed by other authors, ${ }^{31}{ }^{36}$ Salottolo et al showed that in elderly trauma patients, a lactate of $\geq 2.5 \mathrm{mmol} / \mathrm{L}$ led to a 2.62-fold increase in the odds of mortality and that failure to recognise occult hypoperfusion led to an increase in mortality in elderly patients from $12 \%$ to $35 \% .^{1123}$ Venkatesan et al investigated the association between the recorded lactate level and mortality in the hip fracture population and found that $19 \%$ of hip fracture patients showed a lactate of $>3.0 \mathrm{mmol} / \mathrm{L}$ and that these patients showed a mortality 4.5 times greater than those with a lactate of $<3.0 \mathrm{mmol} / \mathrm{L}$ when covariates of interest were adjusted for $(6 \%$ vs $28 \%) .{ }^{30}$

The effect of inadequate resuscitation is exacerbated by the reduced ability of the circulation to deliver oxygen to the end organs with a low serum haemoglobin concentration. Some evidence exists to support this position in the context of anaemia. Anaemia is common among the elderly, affecting $17 \%$ of all patients aged over 65 years, rising to $50 \%$ in patients presenting with hip fracture, in turn leading to compromised patient outcomes and an increased risk of mortality. ${ }^{5} 63738$ The scale of this increased mortality is best described by a meta-analysis of 10 studies investigating patients with hip fracture produced by Potter et al in 2015, showing that preoperative anaemia is associated with an increase in the relative risk of mortality of 1.64 (95\% CI 1.47 to 1.82 ) at all time points beyond 30 days. ${ }^{39}$ Similar effects are seen in elderly 
patients in other surgical cohorts with anaemia causing an increase in mortality of between $6 \%$ and $182 \%$ using a variety of study designs. ${ }^{40-45}$

Adequate resuscitation has a role in reducing the risk bone cement implantation syndrome (BCIS), a condition of ill-defined aetiology associated with an increase in very early postoperative mortality. ${ }^{46}$ This syndrome may occur when the cement that is used to fix in place the femoral stem of patients treated for displaced intracapsular hip fractures is inserted. There is no agreed definition of BCIS; however, it is characterised by hypoxia, hypotension, cardiac arrhythmias, increased pulmonary vascular resistance and cardiac arrest in response to cement or prosthesis implantation at arthroplasty. ${ }^{47}$ It is generally accepted that the pathophysiological process involved is as a result of embolism caused by high intramedullary pressures during cementation. ${ }^{47}$ The risk of BCIS is thought to be reduced by two strategies. One is to alter the surgical field to reduce the risk of embolisation by the use of pulsatile lavage, a suction catheter as well as a retrograde cementation technique. ${ }^{48}$ The second is to ensure adequate fluid resuscitation and maintenance of an adequate blood pressure at the time of cement insertion, failure to do this is associated with excess early death. ${ }^{46}$ The effect of goal-directed intraoperative fluid administration is as yet unclear. Sinclair et al showed in a 40-patient prospective randomised trial that goal-directed restoration of optimal stroke volume reduced length of stay and improved postoperative recovery. ${ }^{49}$ This conclusion was challenged by Price $e t$ al in a 2004 Cochrane Review, which concluded that there was no evidence for using goal-directed intraoperative fluid therapy over a traditional approach. ${ }^{35}$ More recently, this conclusion has been confirmed by Moppett $e t a l$ and Bartha $e t a{ }^{1}$ who both showed in a larger trial that goal-directed fluid therapy does not confer any appreciable benefit. ${ }^{50}$

BCIS does not explain the excess mortality rate in patients with hip fracture. In a large study of over 25000 patients using the Australian Orthopaedic Association National Joint Replacement Registry, Costain et al were able to show that cemented hemiarthroplasty showed equivalent mortality to uncemented prostheses (where BCIS cannot occur) at 7 days and 1 month following surgery, and a reduced mortality at 1 year. ${ }^{52}$ Other mechanisms must have a role in influencing the high mortality observed in these patients.

Preoperative resuscitation using crystalloid, colloid fluids or blood products has the potential to treat hypovolaemia, anaemia and BCIS. ${ }^{29}{ }^{39}{ }^{53-56}$ Crystalloids and colloid fluids do not remain in the circulation for long and fail to address the reduced oxygen carrying capacity,. ${ }^{13} 141617$ Despite the fall in 2,3-diphosphoglycerate (2,3-DPG) concentration in stored blood, the sustained improvement in haemodynamics through the timely administration of blood should lead to improved end organ perfusion. ${ }^{53} 5758$ If a survival advantage exists, then administering appropriate fluid resuscitation preoperatively may prevent deterioration before appropriate treatment can be commenced and prevent irreversible decline in function and the associated increased risk of mortality. The 2015 Cochrane Review by Brunskill et $a \tilde{l}^{9}$ concluded that further research was needed into blood product resuscitation in the preoperative phase of hip fracture care to include frail, unstable and cognitively impaired patients in an effort to improve mortality and functional outcomes. This conclusion is supported by Parker et $a \tilde{l}^{5}$, the FOCUS trial and Potter $e t a l^{39}$, each of which concludes that a trial investigating preoperative blood product resuscitation in patients with hip fracture should take place to investigate potential improvements in the outcomes for these vulnerable patients. ${ }^{56}$

Understandably, it would be reasonable to expect the medical community to be reluctant to administer blood products to patients outside the traditional indications because of the risk of complications and side effects. However, the published refutes these hypotheses and shows that blood administration in elderly adult patients shows no increase in the risk of transfusion-related illness. The Transfusion Requirements in Frail Elderly (TRIFE) study showed no increase in transfusion-related complications in patients with hip fracture when compared with a control group. ${ }^{60}$ This is confirmed by the TITRe 2 trial, the FOCUS trial and by Parker and colleagues who carried out a study in the elderly hip fracture cohort and showed no increase in complications with a protocol-driven transfusion strategy rather than a clinically guided (more restrictive) strategy. ${ }^{5566162}$ Potter et al noted that trials to date, which have shown an increase in morbidity due to transfusion, are influenced by uncontrolled confounding factors, ${ }^{39}$ and Jansen and colleagues describe how the immunological dysfunction attributed to blood administration applies only in massive transfusion. ${ }^{13}$

While the literature is unable to inform the use of blood product in the preoperative resuscitation of elderly adults presenting with hip fracture, it is able to provide an informed foundation on which subsequent investigations can be built. There is a case for interventional trial looking at the effect of preoperative blood product resuscitation in patients with hip fracture with the aim of reducing mortality and improving functional outcomes in answer to their pathophysiology following injury. Equally, there remains sufficient equipoise in using blood products for resuscitation in major trauma for an investigation specific to this patient group to be justified. The use of blood products in this age group is safe and is unlikely to lead to subsequent disease, and the scale of the clinical and socioeconomic burden of this condition is such that an interventional trail with the aims of reducing mortality and morbidity is a reasonable proposal.

\section{CONCLUSION}

The literature contains no evidence of any study that has investigated the effects of preoperative resuscitation in older adult patients presenting with hip fracture, despite 
the high early mortality associated with this injury. Elderly patients are vulnerable to inadequate resuscitation, and from the evidence describing the status of patients with hip fracture and resuscitation in major trauma, we hypothesise that there are advantages to the early appropriate resuscitation of patients with hip fracture. In the absence of any literature or evidence that this is likely to have a deleterious effect, there is sufficient equipoise for the conduct of a randomised controlled trial to establish whether standard care pathways or preoperative resuscitation with blood products in elderly patients with a hip fracture is superior.

Contributors BR and MBK conceived the article. BR conducted the literature search. BR, MRW and MBK all contributed to the production of the manuscript and approved submission.

Provenance and peer review Not commissioned; externally peer reviewed. Data sharing statement No unpublished data is available following this study. Open Access This is an Open Access article distributed in accordance with the Creative Commons Attribution Non Commercial (CC BY-NC 4.0) license, which permits others to distribute, remix, adapt, build upon this work non-commercially, and license their derivative works on different terms, provided the original work is properly cited and the use is non-commercial. See: http://creativecommons.org/ licenses/by-nc/4.0/

(C) Article author(s) (or their employer(s) unless otherwise stated in the text of the article) 2017. All rights reserved. No commercial use is permitted unless otherwise expressly granted.

\section{REFERENCES}

1. Choices NHS. Hip fracture. http://www.nhs.uk/conditions/hipfracture/Pages/introduction.aspx.

2. National hip fracture database. http://www.nhfd.co.uk/20/ hipfractureR.nsf/0/9b0c5ea2e986ff56802577af0046b1df/\$FILE/ Best\%20Practice\%20Tariff\%20User\%20Guide.pdf (accessed 9 Jan 2017).

3. Parker M, Johansen A. Hip fracture. BMJ 2006;333:27-30.

4. National Institute for Health and Care Excellence. Hip fracture management (CG124). 2011 https://www.nice.org.uk/guidance/ cg124 (accessed 9Jan 2017)

5. Penninx BW, Pahor M, Woodman RC, et al. Anemia in old age is associated with increased mortality and hospitalization. J Gerontol A Biol Sci Med Sci 2006;61:474-9.

6. Moppett IK, Parker M, Griffiths R, et al. Nottingham hip fracture score: longitudinal and multi-assessment. $\mathrm{Br} J$ Anaesth 2012;109:546-50.

7. Kannus P, Parkkari J, Sievänen $\mathrm{H}$, et al. Epidemiology of hip fractures. Bone 1996;18(1 Suppl):S57-S63.

8. McGwin G, MacLennan PA, Fife JB, et al. Preexisting conditions and mortality in older trauma patients. J Trauma 2004;56:1291-6.

9. Trauma Audit Research Network- The First Decade. https://www. tarn.ac.uk/Content.aspx?ca=2\&c=36 (accessed 9 Jan 2017).

10. Sterling DA, O'Connor JA, Bonadies J. Geriatric falls: injury severity is high and disproportionate to mechanism. J Trauma 2001;50:116-9.

11. Schulman AM, Claridge JA, Young JS. Young versus old: factors affecting mortality after blunt traumatic injury. Am Surg 2002;68:942-7.

12. Pfeifer R, Tarkin IS, Rocos B, et al. Patterns of mortality and causes of death in polytrauma patients--has anything changed? Injury 2009;40:907-11.

13. Jansen JO, Thomas R, Loudon MA, et al. Damage control resuscitation for patients with Major trauma. BMJ 20091778;338:b17 78;338:b1778

14. McCullough AL, Haycock JC, Forward DP, et al. Early management of the severely injured Major trauma patient. $\mathrm{Br} J$ Anaesth 2014;113:234-41.

15. Dutton RP. Haemostatic resuscitation. Br J Anaesth 2012;109 Suppl 1(Suppl 1):i39-i46.

16. National Institute for Health and clinical excellence. Blood Transfusion 2015 https://www.nice.org.uk/guidance/ng24 (accessed 9 Jan 2017).
17. Joint United Kingdom(UK)Blood transfusion and issue transplantation Services Professional Advisory Committee. Transfusion guidelines. $2016 \mathrm{http}: / /$ transfusionguidelines.org.uk/ (accessed 9Jan 2017).

18. National Institute for Health and Care Excellence. Major trauma:assessment and initial management. 2016 https://www.nice. org.uk/guidance/ng39 (accessed 9Jan 2017)

19. Holcomb JB, Jenkins $D$, Rhee $P$, et al. Damage control resuscitation: directly addressing the early coagulopathy of trauma. J Trauma 2007;62:307-10.

20. Moher D, Liberati A, Tetzlaff J, et al. Preferred reporting items for systematic reviews and meta-analyses: the PRISMA statement. Int $J$ Surg 2010;8:336-41.

21. von Elm E, Altman DG, Egger M, et al. The strengthening the Reporting of Observational studies in Epidemiology (STROBE) statement: guidelines for reporting observational studies. Prev Med 2007;45:247-51.

22. Critical Appraisal Skills Programme (CASP). http://www.casp-uk.net/ (accessed 9Jan 2017).

23. Salottolo KM, Mains $\mathrm{CW}$, Offner $\mathrm{PJ}$, et al. A retrospective analysis of geriatric trauma patients: venous lactate is a better predictor of mortality than traditional vital signs. Scand J Trauma Resusc Emerg Med 2013;21:7.

24. Callaway DW, Shapiro NI, Donnino MW, et al. Serum lactate and base deficit as predictors of mortality in normotensive elderly blunt trauma patients. J Trauma 2009;66:1040-4.

25. Darowski A. The care of patients with fragility fracture. London: British Orthopaedic Association, 2007.

26. British Ortyhopaedic Association Standards for Trauma. http://www. boa.ac.uk/publications/boa-standards-trauma-boasts/. (accessed 9 Jan 2017).

27. Kumar D, Mbako AN, Riddick A, et al. On admission haemoglobin in patients with hip fracture. Injury 2011;42:167-70.

28. Foss NB, Kehlet $\mathrm{H}$. Hidden blood loss after surgery for hip fracture. Bone Joint Surg Br 2006;88:1053-9.

29. Irisson E, Kerbaul F, Parratte S, et al. [Perioperative management based on kinetics of bleeding during total primary arthroplasty]. Ann Fr Anesth Reanim 2013;32:170-4.

30. Venkatesan M, Smith RP, Balasubramanian S, et al. Serum lactate as a marker of mortality in patients with hip fracture: a prospective study. Injury 2015;46:2201-5.

31. Uzoigwe CE, Venkatesan M, Smith R, et al. Serum lactate is a prognostic Indicator in patients with hip fracture. Hip Int 2012;22:580-4

32. Crowl AC, Young JS, Kahler DM, et al. Occult hypoperfusion is associated with increased morbidity in patients undergoing early femur fracture fixation. J Trauma 2000;48:260-7.

33. Okorie ON, Dellinger P. Lactate: biomarker and potential therapeutic target. Crit Care Clin 2011;27:299-326.

34. Vandromme MJ, Griffin RL, Weinberg JA, et al. Lactate is a better predictor than systolic blood pressure for determining blood requirement and mortality: could prehospital measures improve trauma triage? J Am Coll Surg 2010;210:86167-867.

35. Price JD, Sear JW, Venn RM. Perioperative fluid volume optimization following proximal femoral fracture. Cochrane Database Syst Rev 2004;1:Cd003004.

36. del Portal DA, Shofer F, Mikkelsen ME, et al. Emergency department lactate is associated with mortality in older adults admitted with and without infections. Acad Emerg Med 2010;17:260-8.

37. Dong X, Mendes de Leon C, Artz A, et al. A population-based study of hemoglobin, race, and mortality in elderly persons. J Gerontol A Biol Sci Med Sci 2008;63:873-8.

38. Gaskell H, Derry S, Andrew Moore R, et al. Prevalence of anaemia in older persons: systematic review. BMC Geriatr 2008;8:1.

39. Potter LJ, Doleman B, Moppett IK. A systematic review of preoperative anaemia and blood transfusion in patients with fractured hips. Anaesthesia 2015;70:483-500.

40. Vochteloo AJ, Borger van der Burg BL, Mertens B, et al. Outcome in hip fracture patients related to Anemia at admission and allogeneic blood transfusion: an analysis of 1262 surgically treated patients. BMC Musculoskelet Disord 2011;12:262.

41. Baron DM, Hochrieser $\mathrm{H}$, Posch $\mathrm{M}$, et al. Preoperative anaemia is associated with poor clinical outcome in non-cardiac surgery patients. Br J Anaesth 2014;113:416-23.

42. Wu WC, Schifftner TL, Henderson WG, et al. Preoperative hematocrit levels and postoperative outcomes in older patients undergoing noncardiac surgery. JAMA 2007;297:2481-8.

43. Musallam KM, Tamim HM, Richards T, et al. Preoperative anaemia and postoperative outcomes in non-cardiac surgery: a retrospective cohort study. Lancet 2011;378:1396-407. 
44. Landi F, Russo A, Danese P, et al. Anemia status, hemoglobin concentration, and mortality in nursing home older residents. J Am Med Dir Assoc 2007;8:322-7.

45. Saager L, Turan A, Reynolds LF, et al. The association between preoperative Anemia and 30-day mortality and morbidity in noncardiac surgical patients. Anesth Analg 2013;117:909-15.

46. Griffiths R, Parker M. Bone cement implantation syndrome and proximal femoral fracture. $\mathrm{Br} J$ Anaesth 2015;114:6-7.

47. Donaldson AJ, Thomson HE, Harper NJ, et al. Bone cement implantation syndrome. Br J Anaesth 2009;102:12-22.

48. Timperley AJ, Whitehouse SL. Mitigating surgical risk in patients undergoing hip arthroplasty for fractures of the proximal femur. $J$ Bone Joint Surg Br 20092017;91;91 http://www.nrls.npsa.nhs.uk/ resources/?entryid45=59867\&q=0\%c2\%achip\%c2\%ac

49. Sinclair S, James S, Singer M. Intraoperative intravascular volume optimisation and length of hospital stay after repair of proximal femoral fracture: randomised controlled trial. BMJ 1997;315:909-12.

50. Moppett IK, Rowlands M, Mannings A, et al. LiDCO-based fluid management in patients undergoing hip fracture surgery under spinal anaesthesia: a randomized trial and systematic review. $\mathrm{Br} J$ Anaesth 2015;114:444-59.

51. Bartha E, Arfwedson C, Imnell A, et al. Randomised controlled trial of goal-directed haemodynamic treatment in patients with proximal femoral fracture. Br J Anaesth 2013;110:545-53.

52. Costain DJ, Whitehouse SL, Pratt NL, et al. Perioperative mortality after hemiarthroplasty related to fixation method: a study based on the australian orthopaedic association national joint replacement registry. Acta orthopaedica 2011;82:275-81.
53. Kadar A, Chechik O, Steinberg E, et al. Predicting the need for blood transfusion in patients with hip fractures. Int Orthop 2013;37:693-700.

54. Biboulet P, Dadure C, Capdevila X. Postoperative transfusion thresholds: to wait or to anticipate? this is the question. Anaesth Crit Care Pain Med 2015;34:313-4.

55. Parker MJ. Randomised trial of blood transfusion versus a restrictive transfusion policy after hip fracture surgery. Injury 2013;44:1916-8.

56. Carson JL, Terrin ML, Noveck $\mathrm{H}$, et al. Liberal or restrictive transfusion in high-risk patients after hip surgery. $N$ Engl $J$ Med 2011;365:2453-62.

57. Högman CF, Löf H, Meryman HT. Storage of red blood cells with improved maintenance of 2,3-bisphosphoglycerate. Transfusion 2006;46:1543-52.

58. Qu L, Triulzi DJ. Clinical effects of red blood cell storage. Cancer Control 2015;22:26-37.

59. Brunskill SJ, Millette SL, Shokoohi A, et al. Red blood cell transfusion for people undergoing hip fracture surgery.. Cochrane Database Syst Rev 2015;4:Cd009699.

60. Gregersen M, Borris LC, Damsgaard EM. Postoperative blood transfusion strategy in frail, anemic elderly patients with hip fracture: the TRIFE randomized controlled trial. Acta Orthop 2015;86:363-72.

61. Murphy GJ, Pike K, Rogers CA, et al. Liberal or restrictive transfusion after cardiac surgery. N Engl J Med 2015;372:997-1008.

62. Gregersen M, Damsgaard EM, Borris LC. Blood transfusion and risk of infection in frail elderly after hip fracture surgery: the TRIFE randomized controlled trial. Eur J Orthop Surg Traumatol 2015;25:1031-8. 